\title{
Riesgo de suicidio en población pediátrica tratada con antidepresivos: impacto de la alerta de 2003 en la prescripción e investigación en eficacia y seguridad
}

\author{
INMACULADA PALANCA M. ${ }^{1}$, LIDIA FERNÁNDEZ M. ${ }^{1}$, CONSUELO MORANT G. ${ }^{2}$ \\ 1. Unidad de Psiquiatría Infantil. Servicio de Psiquiatría. Hospital Universitario Puerta de Hierro Majadahonda. \\ Madrid. España. \\ 2. Responsable de Epidemiología. Oficina Regional de Salud Mental de la Comunidad de Madrid. España.
}

\begin{abstract}
Suicide risk in pediatric populations treated with antidepressives: impact of the 2003 alert in prescription and research in efficacy and safety

Objective: To analyze the use and research in efficacy and safety of antidepressants in Mayor Depressive Disorder (MDD) in paediatric population after the 2003 international warning from regulatory agencies (EMA, FDA) about an increased suicide risk in children and adolescents treated with antidepressants. Method: A review of the last 10 years publications regarding the prescription trends and efficacy and safety of antidepressants in paediatric depression was carried out. Cochrane and Pub Med databases were used. Results: Since the alert there has been a change in previous ascending trends of antidepressant prescription to children and adolescents with a decrease in their use, as well as an increase in the completed suicide rate in this population. Clinical management and monitoring of these patients have been slightly modified. Since 2004, two studies (escitalopram, citalopram) showed significant efficacy vs placebo for paediatric depression, adding more evidence to previous Fluoxetine results. Three studies show significant efficacy in adolescent MDD but only one with sertraline was designed to detect subgroup age differences. No more evidence of increased suicide risk has been found. Conclusions: Safety warnings from regulatory agencies EMA and FDA about antidepressant use in paediatric population has modified sligthtly clinical practice, with a decreased trend in prescription. More research has been conducted since 2004, adding evidence for efficacy but not for an increased risk of suicide. Methodological flaws of conducted studies after the warnings remain similar to previous research.

(Key words: Adolescents, children, antidepressant agents, depression, suicide risk, warning).

Rev Chil Pediatr 2011; 82 (4): 277-288
\end{abstract}

Trabajo recibido el 03 de febrero de 2011, devuelto para corregir el 21 de abril de 2011, segunda versión el 02 de junio de 2011, aceptado para publicación el 04 de julio de 2011.

Correspondencia a:

Inmaculada Palanca M.

E-mail: ipalanca.hgugm@salud.madrid.org 


\section{RESUMEN}

Objetivos: Analizar el uso de antidepresivos (AD) y la investigación sobre su eficacia y seguridad en población pediátrica con Trastorno Depresivo Mayor (TDM), tras la alerta de las agencias reguladoras European Medicines Agency (EMA) y la americana Food and Drugs Administration (FDA) en 2003 sobre el riesgo de suicidio en menores de 18 años tratados con antidepresivos. Métodos: Se han revisado las publicaciones en los últimos 10 años sobre eficacia y seguridad de los antidepresivos en menores de 18 años, y sobre las prácticas de prescripción de estos fármacos en esta población. Se utilizaron las bases de datos Cochrane y Pub Med. Resultados: Desde la Alerta se ha producido un cambio en la tendencia previa ascendente en la prescripción de antidepresivos a niños y adolescentes, así como un incremento de las tasas de suicidio en esta población. La monitorización de estos pacientes se ha modificado ligeramente. Desde 2004, dos ensayos clínicos (1 citalopram, 1 escitalopram) han obtenido significación estadística en eficacia frente a placebo en niños y adolescentes, sumándose a la evidencia previa para fluoxetina. Tres estudios demuestran eficacia en adolescentes pero sólo uno con sertralina fue diseñado para evaluar separadamente los grupos de edad. No se han encontrado estudios que añadan evidencia sobre el incremento del riesgo de suicidio. Conclusiones: La advertencia de las agencias reguladoras (EMA y FDA) ha modificado levemente la práctica clínica de los profesionales. Se han publicado estudios desde 2004, que aportan nuevos resultados de eficacia pero no más evidencia respecto a su seguridad.

(Palabras clave: Adolescentes, niños, antidepresivos, depresión, riesgo de suicidio, advertencia).

Rev Chil Pediatr 2011; 82 (4): 277-288

\section{Introducción}

El diagnóstico y tratamiento de la depresión en niños y adolescentes es de particular importancia debido a las posibles implicaciones para el funcionamiento en la vida adulta, mucho más allá del ámbito de la salud ${ }^{1}$. La medicación representa una opción de primera línea en el abordaje terapéutico del trastorno depresivo mayor (TDM) moderado-grave y debe valorarse de forma individualizada ${ }^{2-4}$. La alerta que lanzaron la agencias reguladoras europea (EMA) y americana (FDA) en 2003 por el riesgo de suicidio asociado al uso de antidepresivos en menores, dio lugar a un debate internacional entre profesionales del ámbito asistencial e investigadores sobre la adecuación de dicha actuación y las consecuencias de la misma sobre el manejo de la depresión en este grupo de población.

La alerta sobre el riesgo de suicidio en niños y adolescentes tratados con antidepresivos se inició paralelamente en 2003 en Europa y EE.UU. por las conclusiones sobre paroxetina del Comité de Seguridad de Medicamentos del Reino Unido (MHRA); en el arbitraje solicitado por este organismo a la EMA, el Comité de Medicamentos de Uso Humano (CHMP) de esta agencia amplió la revisión sobre eficacia y seguridad al resto de los inhibidores selectivos de la recaptación de la serotonina (ISRS) y a los inhibidores de la recaptación de la serotonina y noradrenalina. En abril de 2005 el CHMP concluyó que en los ensayos clínicos, publicados y no publicados, realizados en menores, los comportamientos suicidas, la hostilidad, comportamientos oposicionistas y ataques de ira eran más frecuentes en menores tratados con atomoxetina, fluoxetina, citalopram, escitalopram, fluvoxamina, paroxetina, sertralina, venlafaxina, duloxetina, mianserina, milnacepram, mirtazapina y reboxetina, que en los tratados con placebo (3,7\% vs $2,5 \%$ respectivamente), recomendando, en la Unión Europea, incluir una advertencia para informar a médicos y padres de estos riesgos. Paralelamente, la FDA llegó a conclusiones similares, decidiendo incluir una advertencia en el etiquetado de todos los antidepresivos y haciendo recomendaciones de seguimiento recogidas en una guía. Como consecuencia de estas alertas, prácticamente todos los países desarrollados tomaron medidas similares.

Previo a la alerta, se observaba una ten- 
dencia creciente en la prescripción de antidepresivos en niños y adolescentes, con porcentajes variables en las distintas publicaciones atribuidos a aspectos metodológicos ${ }^{1,5-7}$. En el Reino Unido se experimentó un incremento anual sostenido hasta la alerta de las agencias reguladoras EMA y $\mathrm{FDA}^{8}$, produciéndose un aumento del 68\% desde 1993 hasta 20025 . En Estados Unidos (EE.UU.), el uso de antidepresivos en niños con un primer episodio depresivo aumentó del 5\% en 1998 al 37\% en $2002^{9}$. En 2002 más de 10 millones de antidepresivos fueron prescritos en niños y adolescentes en EE.UU. ${ }^{10}$; aproximadamente $6 \%$ de las visitas ambulatorias de menores entre 5 y 17 años incluían la prescripción de antidepresivos $^{11}$. En 2009 Skaer y cols, realizaron una encuesta nacional a médicos ambulatorios (NAMCS) estudiando la prescripción de tricíclicos e ISRS (citalopram, fluoxetina, paroxetina y sertralina) en menores entre 5 y 18 años en 3 períodos: 1990-1993, 1994-1997, y 1998-2001. La prescripción de antidepresivos en pacientes diagnosticados de depresión pasó del 44,4\% entre $1990-93$ al 59,3\% entre 19982001. Durante este período, el uso de ISRS aumentó desde un 20,7\% entre 1990-93 hasta un 39,7\% entre 1998-2001, y la prescripción de tricíclicos descendió del 21\% entre 1990-93 hasta un 2,7\% entre 1998-2001. La fluoxetina fue el antidepresivo más prescrito ${ }^{12}$.

En los últimos años, numerosos trabajos científicos han analizado la repercusión que la alerta sobre seguridad de los antidepresivos en niños y adolescentes con trastorno depresivo haya podido tener sobre el uso de antidepresivos y sobre la investigación en este campo.

El objetivo de este trabajo es determinar la tendencia en los últimos años del uso de antidepresivos en población pediátrica, así como analizar si desde las alertas del año 2003 se ha sumado más evidencia de eficacia y seguridad de estos fármacos en niños y adolescentes.

\section{Métodos}

Realizamos una búsqueda de las publicaciones sobre eficacia y seguridad de antidepresivos desde enero de 1990 a octubre de 2010, en Pub Med y Cochrane con las palabras clave: adolescents, children, depression, suicide risk, antidepressive agents, fluoxetine, sertraline, paroxetine, citalopram, escitalopram y venlafaxine. Se seleccionaron todos los ensayos clínicos aleatorizados (ECA) versus placebo y todos los estudios abiertos prospectivos (EAP) realizados con inhibidores selectivos de la recaptación de la serotonina (ISRS) (fluoxetina, paroxetina, sertralina, citalopram y escitalopram), venlafaxina, duloxetina o tricíclicos realizados en muestras de 30 o más sujetos y en población menor de 18 años con diagnóstico de distimia o TDM. Se analizaron las siguientes variables: tamaño y origen de la muestra, edad, diagnóstico, gravedad, duración, inclusión de pacientes con riesgo de suicidio, evaluación del resultado, fármaco y dosis, y resultados de eficacia. Fueron seleccionados 7 artículos publicados antes de la alerta del 2003 y 12 publicados entre 2004 y 2010. De los 19, 11 fueron ECA y 8 EAP. Dos estudios fueron rechazados por el pequeño tamaño muestral $(\mathrm{N}<30)$. A su vez, utilizando las palabras clave: prescribing trends, pediatric depression treatment, psychotropic medication, black box warning y FDA warning. Se efectuó una búsqueda de artículos científicos sobre tendencias de uso y prescripción de antidepresivos en esta población desde 1997 hasta 2010, encontrándose 17 artículos relacionados.

\section{Resultados}

\section{Tendencias de prescripción}

Trascurridos 7 años, podemos preguntarnos qué efecto ha tenido la alerta sobre la prescripción de antidepresivos en población infantojuvenil y cuál ha sido la evolución de las tasas de suicidio, clave en la alerta sobre seguridad.

Distintos trabajos han estudiado la tendencia de la prescripción después de la alerta. Cheung y cols (2008), publicaron los resultados de una encuesta a pediatras realizada en 2005 sobre el uso de antidepresivos: el 72\% eran conscientes de la alerta y de éstos, el 80\% cambiaron su práctica en la prescripción de forma que el 32\% siguieron más estrechamente los pacientes, un $7 \%$ cambiaron de tratamiento 
al menos en un paciente y un $8 \%$ suspendió la medicación por decisión del paciente ${ }^{11}$. Bathia y cols (2008), estudiaron el uso de los antidepresivos en población pediátrica mediante una encuesta autoaplicada a médicos de EE.UU. entre julio y agosto de 2005. Los antidepresivos más prescritos fueron: sertralina $(39,8 \%)$, fluoxetina (19,4\%) y escitalopram (18,5\%). El 96,8\% de los profesionales conocían la alerta, aunque el 76,9\% seguían prescribiendo antidepresivos (99,5\% a adolescentes y 39,2\% a menores de 12 años); no obstante, un 15,5\% reconoció haber disminuido la prescripción en niños y un 36,6\% en adolescentes. El 31,9\% aumentaron la frecuencia de las visitas pero sólo el 7,5\% siguieron la recomendación de la FDA (citas semanales durante el primer mes). El 21,9\% informaron sobre pacientes o cuidadores que se habían negado a tomar antidepresivos, basándose en las advertencias. Al igual que en otros estudios, se detectó un descenso en la prescripción de antidepresivos, aunque la mayoría de los encuestados no seguían las recomendaciones de la FDA por considerar clínicamente justificado el uso de antidepresivos $^{9}$.

Morrato y cols (2008), investigaron el posible impacto de la alerta sobre el manejo clínico, mediante el análisis retrospectivo de una base de datos asistenciales entre 1998 y 2005 con 27370 menores (5-18 años) y 193151 adultos. Ambos grupos, con diagnóstico de TDM en tratamiento, se evaluaron midiendo el cumplimiento de dos instrumentos: el HEDIS (examina el contacto óptimo con salud mental, midiendo la duración del tratamiento las primeras 12 semanas y los 6 primeros meses) y las recomendaciones de la guía de la FDA en 2005 (7 visitas durante los primeros 3 meses de tratamiento). Se concluyó que menos del 5\% de la población infanto-juvenil siguieron las recomendaciones de la FDA antes de la alerta y sólo el $21 \%$ siguieron por completo el criterio de HEDIS, sin cambios significativos tras la alerta. Cumplieron mejor los criterios de HEDIS aquellos pacientes vistos por un psiquiatra (80\%) que los asistidos por un pediatra (60\%) o por un médico de familia (54\%), sin embargo, no hubo diferencias significativas en el cumplimiento de las recomendaciones de la
$\mathrm{FDA}^{13}$. Una limitación del estudio es la diferencia del seguimiento previo y posterior a la alerta (5 y 1,5 años respectivamente), por lo que un mayor seguimiento posterior podría variar los resultados.

Además, en alguno de estos estudios, junto al descenso en la prescripción de antidepresivos a niños y adolescentes, se constató un incremento de las prescripciones desde Servicios de Psiquiatría frente a Atención Primaria, aumentando las listas de espera. En EE.UU., el 44\% del global de pacientes menores de 18 años con depresión, eran atendidos en psiquiatría en 2004, un año después era el 63\% ${ }^{8}$.

\section{Prescripción de antidepresivos y suicidio}

Estudios ecológicos de tendencias históricas objetivaron un incremento sostenido de la prescripción de antidepresivos en menores antes de la advertencia (91\% en EE.UU. y 120\% en Holanda entre 1998-2003) y una disminución de las tasas de suicidio en esta población (33\% en EE.UU. y 31\% en Holanda entre 1998-2003) ${ }^{8,14,15}$. Estudios similares tras la alerta de las agencias reguladoras EMA y FDA han constatado un descenso en la prescripción de antidepresivos (22\% en Holanda y $20 \%$ en EE.UU.) y un incremento en las tasas de suicidio (64\% en menores de 20 años en Holanda con mayor efecto en varones menores de 15 años, $y$ un 14\% en EE.UU en menores de 5-19 años) ${ }^{8,14,16}$. Aunque este tipo de estudios no permiten inferir causalidad, la asociación entre ambos factores es estadísticamente significativa $^{8,14}$. Otros estudios evidencian un descenso de las cifras de suicidio desde los años 90 hasta 2004, y un aumento del $18 \%$ tras la advertencia de la $\mathrm{FDA}^{6,14}$, sugiriendo los autores que este aumento de la tasa de suicidio podría relacionarse con un descenso del $18 \%$ en la prescripción de antidepresivos encontrada en algunos estudios ${ }^{3,9,10}$. Contrariamente, Morrato y cols (2008), no encontraron aumentos significativos de las tasas de suicidio relacionados con la prescripción de antidepresivos ${ }^{13}$.

Spindel y cols (2008), señalaron el riesgo de suicidio 90 días antes y 180 días después de iniciarse el tratamiento (antidepresivos o psicoterapia), sobre una gran base de datos de 
pacientes ambulatorios. Se recogió que el mes anterior al inicio del tratamiento tenía el mayor riesgo de tentativas, sugiriendo que los intentos de suicidio eran una causa para iniciar el tratamiento y no el resultado del mismo ${ }^{3}$. Estudios sobre resultados de autopsias, obtienen que sólo en un 3\% de los jóvenes suicidas tratados en servicios sanitarios públicos se detectaron psicofármacos en plasma ${ }^{17}$. Otros estudios toxicológicos revelan que del $8 \%$ al $20 \%$ tendría antidepresivos en sangre al momento de su muerte ${ }^{17}$, y otros no hallaron niveles en jóvenes supuestamente en tratamiento previo al fallecimiento ${ }^{11}$. Estos resultados indicarían que la mayoría no tomaba su medicación, al menos los días previos al suicidio.

Respecto al incremento cercano al 2\% del riesgo autolítico entre los grupos de antidepresivos y el grupo placebo objetivado en las revisiones de las agencias reguladoras European Medicines Agency (EMA) y la norteamericana Food and Drugs Administration (FDA), algunos autores opinan que no tendría ningún impacto clínico frente a las ventajas del tratamiento, pues un efecto secundario es significativo cuando afecta, al menos, al 10\% de los sujetos, o cuando hay una diferencia del $4 \%$ sobre placebo ${ }^{17}$.

Cabe añadir que los ensayos clínicos sobre suicidio con antidepresivos presentan importantes limitaciones, como la exclusión de pacientes de alto riesgo o con antecedentes de conductas suicidas, tamaño muestral insuficiente, identificación del riesgo basado en 1 ítem de una escala o entrevista, mejora del riesgo suicida no evaluado, etc ${ }^{1,3,17}$.

Uno de los pocos estudios que no excluye pacientes con ideación suicida (estudio TORDIA) concluye que no disminuye significativamente la incidencia de tentativas suicidas cuando se asocia terapia cognitivo conductual al tratamiento con paroxetina, citalopram y velanfaxina ${ }^{18}$.

Por otra parte, varios estudios observacionales publicados indican que la toma de ISRS en niños y adolescentes constituye un factor de protección para el riesgo de suicidio (Barry y cols, 2010 ${ }^{19}$.

Tiihonen y cols (2006), encontraron que el uso de antidepresivos en niños y en adultos estaba asociado a un mayor riesgo de tentativa de suicidio pero a un menor riesgo de suicidio consumado $^{20}$. Los estudios revisados por las agencias reguladoras EMA y FDA tampoco reportaron ningún caso de suicidio consumado $^{21}$.

Atendiendo a esta información, gran parte de la comunidad científica expresa su preocupación porque la alerta sobre el uso de antidepresivos y las advertencias de las agencias reguladores EMA y FDA hayan conllevado un descenso en el uso de un tratamiento eficaz, con el riesgo que supone no tratar a una población altamente vulnerable ${ }^{17,19}$. Otros autores apoyan la idea de una relación causal entre el descenso de uso de antidepresivos y el aumento de las tasas de suicidio entre los jóvenes ${ }^{9,11,12,17}$.

\section{Evidencia de eficacia de los antidepresivos en niños y adolescentes}

Con la revisión efectuada por las agencias reguladoras European Medicines Agency (EMA) y la norteamericana Food and Drugs Administration (FDA) ${ }^{21}$, se concluyó que los datos existentes no aportaban suficiente evidencia para demostrar la eficacia de los siguientes antidepresivos en el tratamiento de la depresión infantojuvenil: paroxetina, sertralina, fluvoxamina, citalopram, escitalopram, atomoxetina, venlafaxina, duloxetina, mianserina, milnacepram, mirtazapina y reboxetina. Sólo para fluoxetina había evidencia suficiente de eficacia y fue el único antidepresivo en el que el balance beneficio/riesgo se evaluó positivamente, y el único recomendado ${ }^{9,21-25}$.

Hemos querido comprobar si en los años posteriores a la alerta se han realizado estudios que aporten nueva información sobre eficacia de antidepresivos en menores de 18 años que permitan replantearse estas conclusiones. En la tabla 1 se detallan las principales características de los 7 estudios publicados desde el año 1990 hasta el año 2003 y los 12 nuevos publicados desde 2004 hasta $2010^{24,26-43}$. De los siete ECA publicados antes de la alerta en 2003, seis comparan fármaco con placebo ${ }^{24,26,27,34,35,42}$ y uno compara paroxetina con imipramina ${ }^{31}$; cuatro de ellos (3 fluoxetina, 1 sertralina) demostraron eficacia 


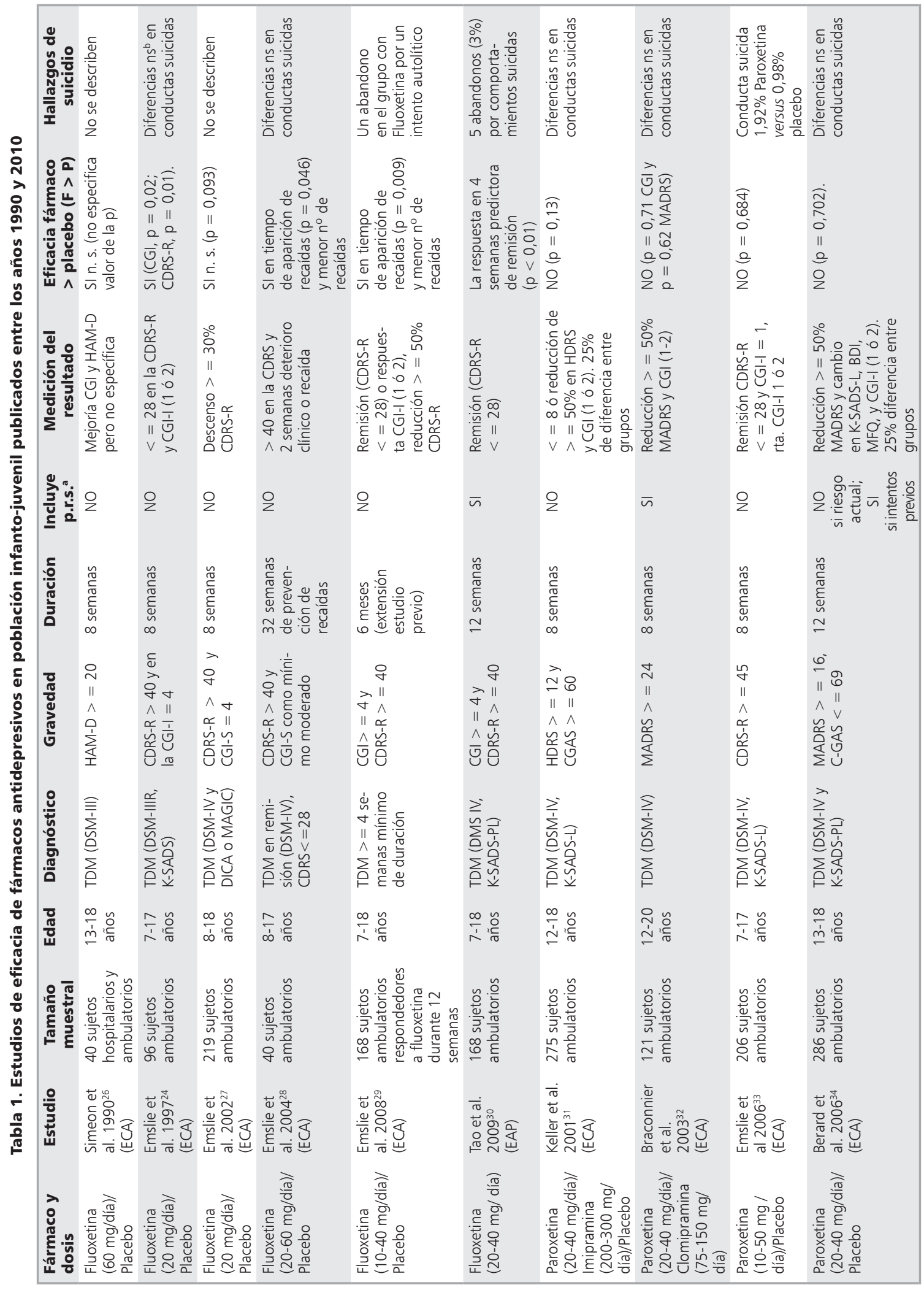




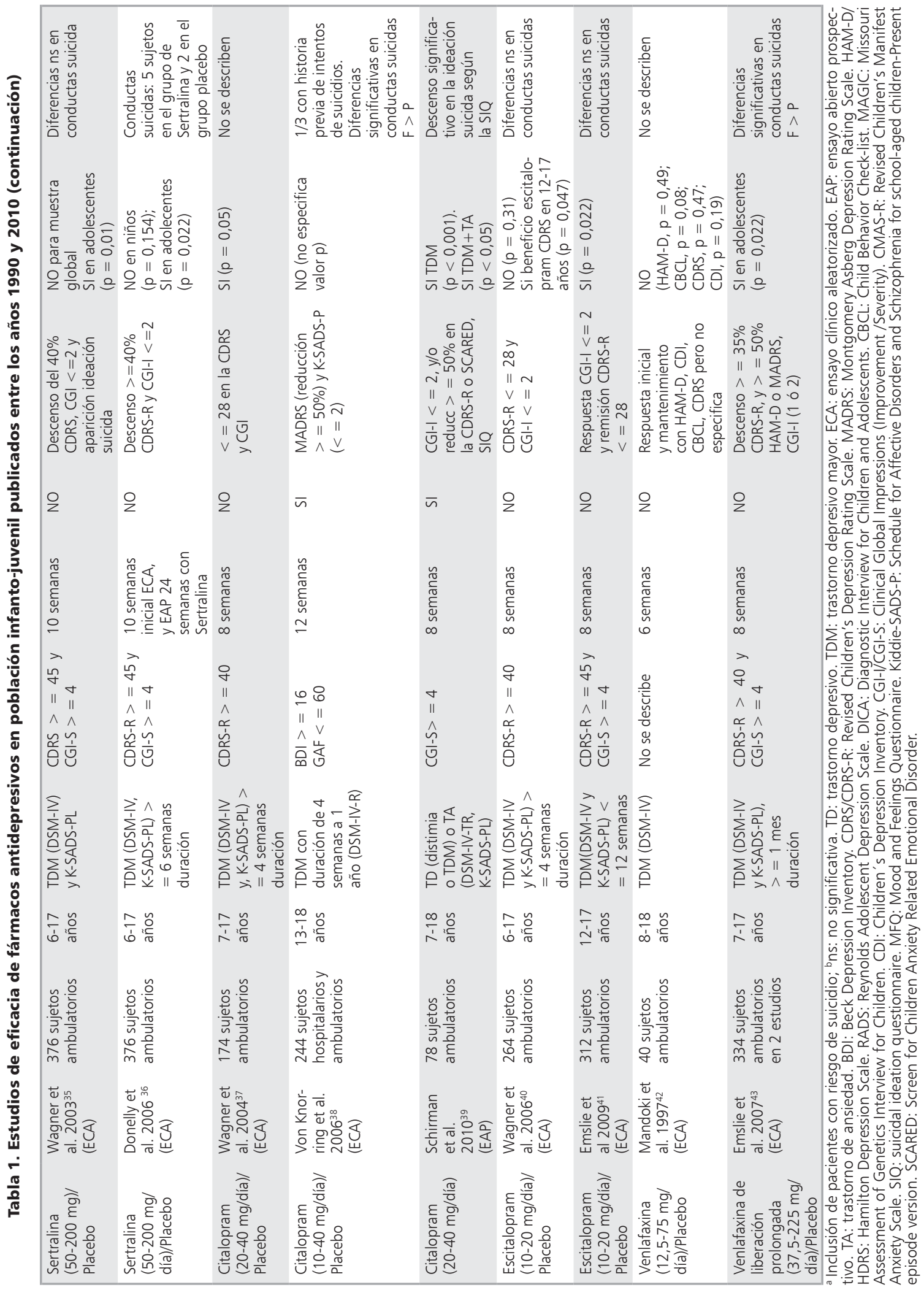


frente a placebo ${ }^{24,26,27,35}$ aunque sólo dos ${ }^{24,35}$ con fluoxetina obtuvieron diferencias estadísticamente significativas, y uno de ellos, con sertralina, únicamente en adolescentes (12-17 años $)^{35}$, pero al no estar diseñado para analizar separadamente los grupos de edad concluyó como negativo. Es relevante la alta respuesta a placebo obtenida en tres estudios (hasta un 30\%) $24,26,35$. Los estudios de Emslie y cols $(2002)^{27}$, para fluoxetina y Keller y cols, para paroxetina $(2001)^{31}$, en que los autores concluyeron mayor eficacia frente a placebo, no fueron aceptados como positivos en el análisis de expertos ${ }^{44}$. Finalmente, sólo Braconnier y cols (2003), incluyeron sujetos con riesgo suicida ${ }^{32}$.

Publicados posteriormente a la alerta (2004-2010), revisamos diez ECA (2 fluoxetina $^{28,29}, 2$ paroxetina ${ }^{33,34}, 1$ sertralina $^{36}, 2$ citalopram $^{37,38}, 2$ escitalopram ${ }^{40,41}, 1$ venlafaxina ${ }^{43}$ ) y dos EAP (1 fluoxetina, 1 citalopram $)^{30,39}$. Dos ECA son estudios de prevención de recaídas en los que Emslie y cols (2004, 2008), demostraron la mayor eficacia de fluoxetina frente a placebo $^{28,29}$.

De los ocho ECA realizados en fase aguda, dos (1 citalopram, 1 escitalopram) demostraron mayor eficacia versus placebo con significación estadística ${ }^{37,41}$. Donelly y cols (2006), en un estudio diseñado para diferenciar resultados por grupos de edad, obtuvieron significativamente mayor respuesta en el mantenimiento con sertralina versus placebo, aunque sólo en adolescentes (12-17 años) ( $\mathrm{p}=$ $0,022)^{36}$. Aunque los estudios de Wagner y cols (2006), con escitalopram y de Emslie y cols (2007), con venlafaxina también sugieren mayor eficacia frente a placebo $(\mathrm{p}=0,047 \mathrm{y} \mathrm{p}=$ 0,022 respectivamente) en este grupo de edad, no fueron diseñados a priori para el análisis de las diferencias de resultados entre niños y adolescentes ${ }^{40,43}$.

Respecto a los EAP, tanto Tao y cols (2009) con el estudio de fluoxetina como predictor de remisión a las 4 semanas $^{30}$, como Schirman y cols (2010), en el estudio con citalopram ${ }^{39}$ demuestran eficacia en esta población; en este último, además, se observó un descenso en la ideación suicida según la SIQ (suicidal ideation questionnaire $)(\mathrm{p}=0,054)^{39}$.
Sólo tres estudios posteriores a la alerta incluyeron sujetos con riesgo de suicidio30, 38,39 y uno (Berard y cols, 2006) incluyó pacientes con conductas suicidas previas ${ }^{34}$.

En resumen, de los 14 ECA seleccionados entre 1990 y 2010, solo 1 publicado previamente a las alertas 2003 de Seguridad (fluoxetina) ${ }^{24}$ y dos con posterioridad a las mismas (1 citalopram, 1 escitalopram) $)^{37,41}$, aportan evidencias significativas de mayor eficacia versus placebo en el tratamiento en fase aguda del trastorno depresivo mayor en población infanto-juvenil y el estudio de Sertralina de Donelly y cols. (2006) también demostró eficacia en el grupo de edad comprendido entre 12-17 años ${ }^{36}$.

\section{Discusión}

Tras la revisión realizada observamos que, respecto a la evidencia de eficacia de los antidepresivos en el tratamiento del TDM en población infantojuvenil, ha habido una mayor publicación de estudios tras la alerta (siete versus doce respectivamente). Sin embargo, además de corroborarse una alta respuesta a placebo $^{24,26,27,35}$, mayor que en adultos, no se han superado algunas limitaciones metodológicas, como la exclusión de población con sintomatología depresiva grave o riesgo de suicidio establecido. Esto hace pensar que las depresiones más graves, con etiología más biológica y donde la respuesta al fármaco frente a placebo puede ser mayor, siguen quedando fuera de los ensayos, sesgando el resultado.

Otro punto a destacar es que las publicaciones encontradas se centran en ISRS e IRSN, en parte porque son los antidepresivos a los que iban dirigidos las alertas fundamentalmente. No obstante, esto supone una limitación a la hora de la interpretación de los resultados, debido a la falta de ensayos clínicos focalizados en otro tipo de antidepresivos.

La fluoxetina sigue siendo el único antidepresivo con indicación aprobada por la European Medicines Agency (EMA) para el tratamiento de la depresión infantil y es el único antidepresivo con un balance beneficio/riesgo positivo según las Agencias Reguladoras European Medicines Agency (EMA) y la nor- 
teamericana Food and Drugs Administration (FDA $)^{19,21-25}$. En algunas revisiones sistemáticas $^{1,44}$, se plantea duramente si esta conclusión para fluoxetina se debe a una mejor calidad de los estudios de fluoxetina más que a diferencias reales de eficacia con el resto de ISRS. Es de destacar, que las tasas de respuesta a los diferentes antidepresivos analizados, es similar en los estudios realizados, alrededor del $70 \%$, siendo lo que establece o no la significación estadística las diferencias en las respuestas a placebo. Este hecho constituiría un argumento a favor del uso en un rango más amplio de los distintos ISRS en la depresión infantil y adolescente. Desde 2004, nuevos datos de eficacia con citalopram y escitalopram avalarían su uso en la depresión infantil y adolescente. En adolescentes, también el escitalopram tiene aprobada recientemente indicación para el TDM por la norteamericana Food and Drugs Administration (FDA). Igualmente, en los estudios realizados, parece observarse una mejor respuesta global a los antidepresivos en adolescentes que en niños, pero al no haber sido diseñados para discriminar diferencias por grupos etarios, las conclusiones, excepto para sertralina, siguen siendo negativas.

En cuanto a la relación del tratamiento antidepresivo en menores con TDM y el riesgo de suicidio, a la luz de los resultados de nuestra revisión, no podemos concluir una relación causal. Por un lado, están las tendencias ascendentes de los suicidios en población infantojuvenil paralelamente al descenso de las prescripciones $8,14,16$ y los estudios que demuestran niveles mínimos o nulos de antidepresivos en plasma en pacientes suicidas que se suponían en tratamiento ${ }^{11,17}$, lo cual indica que podían estar tomando dosis subterapéuticas o haberlo abandonado, o aquellos otros estudios en los que se demuestra el tratamiento farmacológico como factor protector frente al suicidio consumado, aunque pueda aumentar la frecuencia de conducta autolítica. Por otro lado, la mayor incidencia de estas conductas suicidas observada en algunos estudios hace necesario mantener la alerta. Así pues, si los antidepresivos son realmente eficaces en la disminución del riesgo de suicidio por la mejora de la sintomatología depresiva y a la vez existe un incremento del riesgo de conductas suicidas en pacientes tratados con estos fármacos, es bastante probable que existan otras variables relacionadas y no sólo el efecto químico del antidepresivo. Por otro lado, los estudios realizados tras la alerta, siguen adoleciendo de importantes limitaciones metodológicas para el estudio del suicidio.

Por último, en cuanto a los posibles cambios en la práctica clínica de los profesionales que se pudieran derivar de las advertencias de las Agencias reguladoras European Medicines Agency (EMA) y la norteamericana Food and Drugs Administration (FDA), esta revisión muestra que la tendencia al aumento de la prescripción de antidepresivos en la población infantojuvenil se vio parcialmente alterada por las alertas de seguridad en el año 2003, con una disminución sostenida inmediata posterior. En cuanto a las prácticas de manejo clínico de los pacientes, según los estudios publicados, aunque los profesionales conocían las advertencias, no modificaron significativamente las pautas de manejo terapéutico de los pacientes ${ }^{9,13}$

Esto lleva a plantearse la discordancia que puede darse entre la práctica clínica de los profesionales y el monto de evidencia científica que la avala, incitando a una reflexión sobre las razones para ello, tanto en el sentido del freno que la confrontación con consecuencias legales de prescribir un fármaco no aprobado y publicitado como de riesgo suicida tienen, al menos temporalmente, para el profesional que lo prescribe, como en el sentido de que posiblemente la escasez de estudios bien diseñados en población pediátrica obligue a los profesionales a una práctica más basada en la extrapolación de resultados de adultos que en la evidencia científica para población pediátrica.

Así pues, el debate de los profesionales médicos entre prescribir según su experiencia clínica y la información científica accesible o de acuerdo a las normas de las autoridades reguladoras, permanece abierto. La limitada investigación sobre psicofármacos en esta población por las dificultades que conlleva, y la escasez de estudios metodológicamente fiables, hacen que los profesionales otorguen a su propia experiencia un valor que puede entrar en conflicto con lo que se dicta desde el ámbito 
normativo. En este sentido, es necesario que la nueva normativa, tanto de la FDA como de la EMA, para promover la investigación farmacológica en menores, contribuya a incrementar la evidencia científica del efecto de los psicofármacos en las patologías mentales infantojuveniles.

\section{Conclusiones}

1- Se ha invertido la tendencia previa a la alerta de las Agencias reguladoras European Medicines Agency (EMA) y la norteamericana Food and Drugs Administration (FDA), con un descenso del uso de antidepresivos y un incremento de suicidio consumado en niños y adolescentes en Europa y EE.UU.

2- La advertencia de las Agencias reguladoras (EMA y FDA) ha modificado levemente la práctica clínica de los profesionales en el seguimiento de los menores de 18 años en tratamiento antidepresivo, con cumplimiento parcial de las recomendaciones en este sentido.

3- Las limitaciones metodológicas de los ECA posteriores a la alerta en cuanto al estudio del suicidio son similares a las que presentan los estudios previos.

4- Aunque no ha habido modificaciones posteriores a las alertas, según las últimas investigaciones, los beneficios del uso de antidepresivos parecen superar a los riesgos, y algunos de los ECA publicados con posterioridad a las mismas han aportado nuevos resultados de eficacia para citalopram, escitalopram y sertralina en adolescentes, aunque tampoco sean concluyentes respecto al riesgo suicida.

5- La alta utilización de antidepresivos en menores de 18 años contrasta con los escasos estudios con evidencia de eficacia.

6- Es necesario realizar mejoras metodológicas en los estudios que permitan determinar qué antidepresivos son más eficaces para tratar qué subgrupos de pacientes (edad, comorbilidad, etc), aportando mayor evidencia científica a la práctica clínica.

7- La prescripción de otros antidepresivos dis- tintos de fluoxetina, el único aprobado para la depresión en menores de 18 años, sería "off-label", informando en todo momento al paciente $\mathrm{o}$ al tutor legal de este hecho y dejándolo reflejado en la historia clínica.

8- El tratamiento antidepresivo está indicado en niños y adolescentes con trastorno depresivo mayor moderado-grave. La fluoxetina es el único antidepresivo con indicación aprobada en niños por las Agencias Reguladoras Americana y Europea. También existe evidencia de eficacia frente a placebo para citalopram y escitalopram, este último aprobado por la FDA en Adolescentes.

9- El tratamiento farmacológico de la depresión infantil debe formar parte de un tratamiento integral en el que otro tipo de intervenciones de apoyo psicosocial e intervenciones psicoterapéuticas más o menos estructuradas deben estar siempre presentes.

\section{Recomendaciones para el tratamiento de la depresión infantil y adolescente}

Ante una depresión en edad infantil o adolescente debe evaluarse en primer lugar la gravedad de la misma y la limitación funcional que está imponiendo.

En los casos leves-moderados deben contemplarse inicialmente intervenciones de observación activa "watchful waiting" que incluye la demostración de empatía, el reconocimiento de las dificultades que conlleva el estar deprimido y reasegurar que la recuperación es posible, así como la intervención sobre factores estresores.

Si en 2-4 semanas no mejora, deben implementarse tratamientos psicoterapéuticos por un período no inferior a 3 meses. El que ha aportado más evidencia de eficacia es el Tratamiento Cognitivo Conductual en sesiones semanales durante un mínimo de 6 semanas.

A las 4 semanas del inicio de la intervención psicoterapéutica, si no se observa mejoría, así como en los casos moderados-graves, se debe iniciar tratamiento farmacológico con fluoxetina. Es conveniente que quede reflejado en la historia clínica que la familia/tutores legales y el paciente, si es mayor de 16 años 
(mayoría de edad sanitaria), han sido informados de los beneficios y riesgos de éste fármaco. Igualmente deben ser informados de las graves consecuencias del no tratamiento farmacológico (deterioro funcional, riesgo de suicidio).

Como alternativa, citalopram, escitalopram y sertralina pueden ser usados en adolescentes. El tratamiento farmacológico debe mantenerse en prevención de recaídas en casos moderados-graves.

\section{Referencias}

1.- Harrington L: Affective Disorders. En: Child and Adolescent Psychiatry.Cuarta edición.Oxford: Blackwell Science 2003; 486-510.

2.- Recomendaciones Farmacoterapéuticas en Salud Mental. Recomendaciones para la valoración y tratamiento de la depresión infanto-juvenil. Dirección General de Farmacia y Productos Farmacéuticos. Consejería de Sanidad y Consumo. Comunidad de Madrid. Febrero 2006. $n^{\circ} 3$. También en www.madrid.org.

3.- Spindel C, Gabbay V, Coffey B: Adolescent major depression: challenges to treatment. J Child Adolesc Psychopharmacol 2008; 18: 293-6.

4.- National Institute for Health and Clinical Excellence: Depression in children and young people. Identification and management in primary, community and secondary care. Clinical Guideline 28.www.nice.org.uk/CG028.

5.- Wong ICK, Murray ML, Camillero-Novak D, et al: Increased prescribing trends of paediatric psychotropic medications. Arch Dis Child 2004; 89: 1131-2.

6.- Olfson M, Blanco C, Liu L, et al: National trends in the outpatient treatment of children and adolescents with antipsychotic drugs. Arch Gen Psychiatry 2006; 63: 679-85.

7.- Rani F, Murria ML, Byrne PJ, et al: Epidemiologic features of antipsychotic prescribing to children and adolescents in Primary Care in the United Kingdom. Pediatrics.2008;121:1002-1009 Harrington L. Affective Disorders. En: Child and Adolescent Psychiatry. Cuarta edición.Oxford: Blackwell Science 2003; 486-510.

8.- Nemeroff $C B$, Kaladi $A$, Keller $M B$, et al: Impact of Publicity Concerning Pediatric Suicidality Data on Physician Practice Patterns in the United States. Arch Gen Psychiatry 2007; 64: 466-72.

9.- Bhatia SK, Rezac AJ, Vitiello B, et al: Antidepressant Prescribing Practices for the Treatment of Children and Adolescents. J Child Adolesc Psychopharmacol 2008; 18: $70-80$.
10.- Antonuccio D: Treating depressed children with antidepressants: more harm than benefit? J Clin Psychol Med Settings 2008; 15: 92-7.

11.- Cheung A, Sacks D, Dewa CS, et al: Pediatric prescribing Practices and the FDA Black-box Warning on Antidepressants. J Dev Behav Pediatr 2008; 29: 213-5.

12.- Skaer TL, Sclar DA, Robison LM: Trends in prescriptions for antidepressant pharmacotherapy among US children and adolescents diagnosed with depression, 1990 through 2001: an assessment of accordance with treatment recommendations from the American Academy of Child and Adolescent Psychiatry. Clin Ther 2009; 31: 1478-87.

13.- Morrato EH, Libby AM, Orton HD, et al: Frequency of provider contact after FDA advisory on risk of pediatric suicidality with SSRIs. Am J Psychiatry 2008; 165: 4250.

14.- Goodyer IM, Dubicka B, Wilkinson P, et al: A randomised controlled trial of cognitive behaviour therapy in adolescents with major depression treated by selective serotonin reuptake inhibitors. The ADAPT trial. Health Technol Assess 2008; 12: 3-4, 9-60.

15.- Grunebaum MF, Ellis SP, Li S, et al: Antidepressants and Suicide in United States, 1985-1999. J Clin Psychiatry 2004; 65: 1456-62.

16.- Libby AM, Brenda DA, Morato EH, et al: Decline in treatment of pediatric depresión Ander FDA advisory on risk of paediatric suicidality with SSRI. Am J Psychiatry 2006; 163: 41-7.

17.- Silva H, Martínez JC: ¿Es efectivo que los antidepresivos aumentan el riesgo de suicidio? Rev Med Chile 2007; 135: 1195-201.

18.- Brent D, Emslie G, Clarke G, et al: Switching to another SSRI or to venlafaxine with or without cognitive behavioral therapy for adolescents with SSRI-resistant depression: the TORDIA randomized controlled trial. JAMA 2008; 299: 901-13.

19.- Barry CL, Busch SH: News Media Coverage of FDA Warnings on Pediatric Antidepressant Use and Suicidality. Pediatrics 2010; 125: 88-95.

20.- Tiihonen J, et al: Antidepressants and the Risk of Suicide, Attempted Suicide, and Overall Mortality. Archives of General Psychiatry 2006; 63: 1359-67.

21.- European Medicines Agency Press office. European Medicines Agency finalises review of antidepressants in children and adolescents. EMEA/CHMP/ 128918/2005 corr (25 April 2005). En http://www. emea.eu.int/pdfs/human/ press/pr/12891805en.pdf (Consulta 11/9/2004).

22.- Bridge JA, Iyengar S, Salary CB, et al: Clinical Respon- 
se and Risk for reported suicidal ideation and suicide attempts in pediatric antidepressant treatment. A Metaanalysis of randomized controlled trials. JAMA 2007; 297: 1683-96.

23.- March J, Silva S, Petrycki S, et al: Treatment for Adolescents with Depression Study (TADS) Team. Fluoxetine, cognitive-behavioral therapy and their combination for adolescents with depression: Treatment for Adolescents with depression study (TADS) randomized controlled trial. JAMA 2004; 292: 807-20.

24.- Emslie GJ, Rush AJ, Weinberg WA, et al: A doubleblind, randomized, placebo-controlled trial of fluoxetine in children and adolescents with depression. Arch Gen Psychiatry 1997; 54: 1031-7.

25.- Busch SH, Barry CL: Pediatric Depression Treatment in the Aftermath of the Black Box Warning: Implications for Prescription Drug Policy.Health Aff (Millwood). 2009; 28: 724-33.

26.- Simeon JG, Dinicola VF, Ferguson HB, et al: Adolescent depression: a placebo-controlled fluoxetine treatment study and follow-up. Prog Neuropsychopharmacol Biol Psychiatry 1990; 14: 791-5.

27.- Emslie GJ, Heiligenstein JH, Wagner KD, et al: Fluoxetine for acute treatment of depression in children and adolescents: a placebo-controlled, randomized clinical trial. J Am Acad Child Adolesc Psychiatry 2002; 41: 1205-15.

28.- Emslie GJ, Heiligenstein JH, Hoog SL, et al: Fluoxetine treatment for prevention of relapse of depression in children and adolescents: a double-blind, placebo-controlled study. J Am Acad Child Adolesc Psychiatry 2004; 43: 1397-405.

29.- Emslie GJ, Kennard BD, Mayes TL, et al: Fluoxetine versus placebo in preventing relapse of major depression in children and adolescents. Am J Psychiatry 2008; 165: 459-67.

30.- Tao R, Emslie G, Mayes $T$, et al: Early prediction of acute antidepressant treatment response and remission in pediatric major depressive disorder. J Am Acad Child Adolesc Psychiatry 2009; 48: 71-8.

31.- Keller MB, Ryan ND, Strober M, et al: Efficacy of paroxetine in the treatment of adolescent major depression: a randomized, controlled trial. J Am Acad Child Adolesc Psychiatry 2001; 40: 762-72.

32.- Braconnier A, Le Coent R: Paroxetine versus clomipramine in adolescents with severe major depression: a double-blind, randomized, multicenter trial. J Am Acad Child Adolesc Psychiatry 2003; 42: 22-9.

33.- Emslie GJ, Wagner KD, Kutcher $S$, et al: Paroxetine treatment in children and adolescents with major depressive disorder: a randomized, multicenter, doubleblind, placebo-controlled trial. J Am Acad Child Adolesc Psychiatry 2006; 45: 709-19.

34.- Berard R, Fong R, Carpenter DJ, et al: An international, multicenter, placebo-controlled trial of paroxetine in adolescents with major depressive disorder. J Child Adolesc Psychopharmacol 2006; 16: 59-75.

35.- Wagner KD, Ambrosini P, Rynn M, et al: Efficacy of sertraline in the treatment of children and adolescents with major depressive disorder: two randomized controlled trials. JAMA 2003; 290: 1033-41.

36.- Donnelly CL, Wagner KD, Rynn M, et al: Sertraline in children and adolescents with major depressive disorder. J Am Acad Child Adolesc Psychiatry 2006; 45: 1162-70.

37.- Wagner KD, Robb AS, Findling RL, et al: A randomized, placebo-controlled trial of citalopram for the treatment of major depression in children and adolescents. Am J Psychiatry 2004; 161: 1079-83.

38.- Von Knorring AL, Olsson GI, Thomsen PH, et al: A randomized, double-blind, placebo-controlled study of citalopram in adolescents with major depressive disorder. J Clin Psychopharmacol 2006; 26: 311-5.

39.- Schirman S, Kronenberg S, Apter A, et al: Effectiveness and tolerability of citalopram for the treatment of depression and anxiety disorders in children and adolescents: an open-label study. J Neural Transm 2010; 117: 139-45.

40.- Wagner KD, Jonas J, Findling RL, et al: A double-blind, randomized, placebo-controlled trial of escitalopram in the treatment of pediatric depression. J Am Acad Child Adolesc Psychiatry 2006; 45: 280-8.

41.- Emslie GJ, Ventura D, Korotzer A, et al: Escitalopram in the treatment of adolescent depression: a randomized placebo-controlled multisite trial. J Am Acad Child Adolesc Psychiatry 2009; 48: 721-9.

42.- Mandoki MW, Tapia MR, Tapia MA, et al: Venlafaxine in the treatment of children and adolescents with major depression. Psychopharmacol Bull 1997; 33: 149-54.

43.- Emslie GJ, Findling RL, Yeung PP, et al: Venlafaxine ER for the treatment of pediatric subjects with depression: results of two placebo-controlled trials. J Am Acad Child Adolesc Psychiatry 2007; 46: 479-88.

44.- Jureidini J, Doecke C, Mansfield P, et al: Efficacy and safety of antidepressants for children and adolescents. BMJ 2004; 328: 879-83.

45.- Cheung AH, Emslie GJ,Mayes TL: Review of the efficacy and safety of antidepressants in youth depression. J Child Psychol Pychiatry 2005; 46: 735-54. 